\title{
REVIEW ARTICLE \\ Resistant Gram-negative infections in the outpatient setting in Latin America
}

\author{
M. J. C. SALLES ${ }^{*}$, J. ZURITA ${ }^{2}$, C. MEJÍA ${ }^{3}$ AND M. V. VILLEGAS ${ }^{4}$ on behalf of the \\ Latin America Working Group on Bacterial Resistance $\dagger$ \\ ${ }^{1}$ Internal Medicine Department, Santa Casa de São Paulo School of Medicine, São Paulo, Brazil \\ ${ }^{2}$ Hospital Vozandes, Facultad de Medicina, Pontificia Universidad Católica del Ecuador, Quito, Ecuador \\ ${ }^{3}$ Infectious Diseases Unit, Hospital Roosevelt, Guatemala City, Guatemala \\ ${ }^{4}$ Bacterial Resistance Group, International Center for Medical Research and Training (CIDEIM), \\ Cali, Colombia
}

Received 16 April 2013; Final revision 25 June 2013; Accepted 10 July 2013; first published online 7 August 2013

\section{SUMMARY}

Latin America has a high rate of community-associated infections caused by multidrug-resistant Enterobacteriaceae relative to other world regions. A review of the literature over the last 10 years indicates that urinary tract infections (UTIs) by Escherichia coli, and intra-abdominal infections (IAIs) by E. coli and Klebsiella pneumoniae, were characterized by high rates of resistance to trimethoprim/sulfamethoxazole, quinolones, and second-generation cephalosporins, and by low levels of resistance to aminoglycosides, nitrofurantoin, and fosfomycin. In addition, preliminary data indicate an increase in IAIs by Enterobacteriaceae producing extended-spectrum $\beta$-lactamases, with reduced susceptibilities to third- and fourth-generation cephalosporins.

Primary-care physicians in Latin America should recognize the public health threat associated with UTIs and IAIs by resistant Gram-negative bacteria. As the number of therapeutic options become limited, we recommend that antimicrobial prescribing be guided by infection severity, established patient risk factors for multidrug-resistant infections, acquaintance with local antimicrobial susceptibility data, and culture collection.

Key words: Drug resistance, Gram-negative, intra-abdominal infection, Latin America, outpatient, urinary tract infection.

\section{INTRODUCTION}

Antibacterial resistance in clinically important Gramnegative pathogens is transmitted easily among individuals in various community settings via water,

\footnotetext{
* Author for correspondence: Dr M. J. C. Salles, Infectious Diseases Clinic, Internal Medicine Department, Santa Casa de São Paulo School of Medicine, Rua Dr Cesareo Mota Jr 112, Hospital da Irmandade da Santa Casa, São Paulo, 01221-020, Brazil.

(Email: mcsalles@osite.com.br)

$\dagger$ Members of the Latin America Working Group on Bacterial Resistance are listed in the Appendix.
}

sanitation, hygiene, and food pathways [1-6]. In both urban and rural settings, there are many means by which resistant pathogens are disseminated, including human migration, overcrowding, chemical pollution, waste water, and untreated groundwater [3,5-9]. An important mode of transmission of resistant Gramnegative bacteria includes animal vectors, as observational data have clearly shown a similarity in the resistance genes and enterotoxins of Escherichia coli isolated from companion animals and horses, and extended-spectrum $\beta$-lactamase (ESBL)-producing isolates described in humans [10]. Faecal carriage 
of antibiotic-resistant pathogens in humans and animals predisposes to further contamination of food and water supplies to complete a transmission cycle $[4,11-$ $16]$.

Latin America fulfils all of the above-mentioned criteria required to drive the spread of antibacterial drug resistance. As in other world regions, high antibacterial use and misuse (e.g. inappropriate drug selection, suboptimal dosing, poor patient adherence) may also drive bacterial resistance in Latin America [7, 17]. Consequently, for many pathogens, rates of antimicrobial resistance in Latin America appear to be high relative to other regions of the world $[8,18]$.

The unique set of medical, societal, and ecological circumstances in Latin America has underpinned a dynamic epidemiology of Gram-negative infections in the outpatient setting over the last 15 years. In particular, community-associated infections caused by multidrug-resistant Enterobacteriaceae and other Gram-negative bacteria are an important public health concern [19-25]. Foremost of the pathogens causing concern are strains of E. coli-producing ESBLs, which are an important cause of urinary tract infection (UTI) and intra-abdominal infection (IAI) sometimes associated with bacteraemia [19-21, 23, 26, 27]. Because ESBL-producing bacteria now cause many infections in the community setting, the medical community is increasingly reliant on multilevel microbial surveillance to inform treatment decisions, identify major problems, and guide adequate control measures [19-22, 26, 28].

This narrative review stems from the 10th Meeting of the Latin America Working Group on Bacterial Resistance in São Paulo, Brazil, 20-21 May 2012, at which the Working Group members reflected on the increasing recognition of the clinical importance of multidrug-resistant Gram-negative infections in the community setting in Latin America. Using primary data from published studies and policy documents, the aim of this review is to report on the epidemiology of Gram-negative bacteria isolated in Latin America over the last 10 years, describe the importance of detection in outpatients, and consider the implications for prescribing decisions.

\section{METHODS}

In order to review the published clinical data relating to UTIs and IAIs due to Gram-negative pathogens in the community setting of Latin America, a systematic search of the biomedical literature was conducted. The title/abstract fields of Pubmed were searched, limited by the dates 1 January 2005 to 12 November 2012, for articles using the following terms and Boolean logic: ('Latin America' OR 'South America' OR 'Central America' or Mexico or Guatemala or Honduras or Nicaragua OR 'Costa Rica' OR Cuba OR 'Dominican Republic' OR Panama OR Colombia or Venezuela OR Guyana OR Suriname oR 'French Guiana' OR Brazil OR Ecuador OR Peru OR Bolivia OR Paraguay OR Uruguay or Chile oR Argentina) and ('Gram-negative infection' OR 'Gram-negative pathogen' OR 'Gramnegative bacilli' OR 'Escherichia coli' OR 'Klebsiella pneumoniae' OR 'Proteus mirabilis' oR Citrobacter OR Serratia OR 'urinary tract infection' OR 'intraabdominal infection'). In addition, we searched the Scientific Electronic Library Online (SciELO), which publishes health science data specifically from Latin America. Searches on SciELO were limited to policy statements. A small number of additional references were identified from the reference lists of published articles.

The titles and abstracts of all references obtained in the search were screened by the authors. We included all original research articles that reported information on the: (1) susceptibilities of Gram-negative pathogens causing UTIs and IAIs in Latin American primary care; (2) proportion of Gram-negative clinical isolates harbouring ESBL genes or expressing ESBLs; and (3) presence of circulating ESBLs and first reports of novel ESBLs. Only studies reporting data for $>40$ isolates were selected for assessing drug resistance.

\section{EPIDEMIOLOGY}

\section{Overview}

ESBLs are found predominantly in Klebsiella spp. and E. coli, but have also been described in other genera of Enterobacteriaceae including species of Citrobacter, Serratia, Proteus, Salmonella, and Enterobacter [29, 30]. Until the turn of the century, Klebsiella pneumoniae harbouring Temoneira (TEM)-type and sulfhydryl variable (SHV)-type ESBLs were prevalent in the nosocomial setting only, and cefotaxime-resistant (CTX-M) $\beta$-lactamase-producing organisms were rarely isolated (Fig. 1) [30-32]. However, in the first years of this century, Latin America became the first continent where CTX-M variants began to displace TEM and SHV variants as the most common type of ESBL, mainly in E. coli [31]. CTX-M-type ESBLs share only $40 \%$ homology with TEM or SHV enzymes and are considered unrelated [30]. 


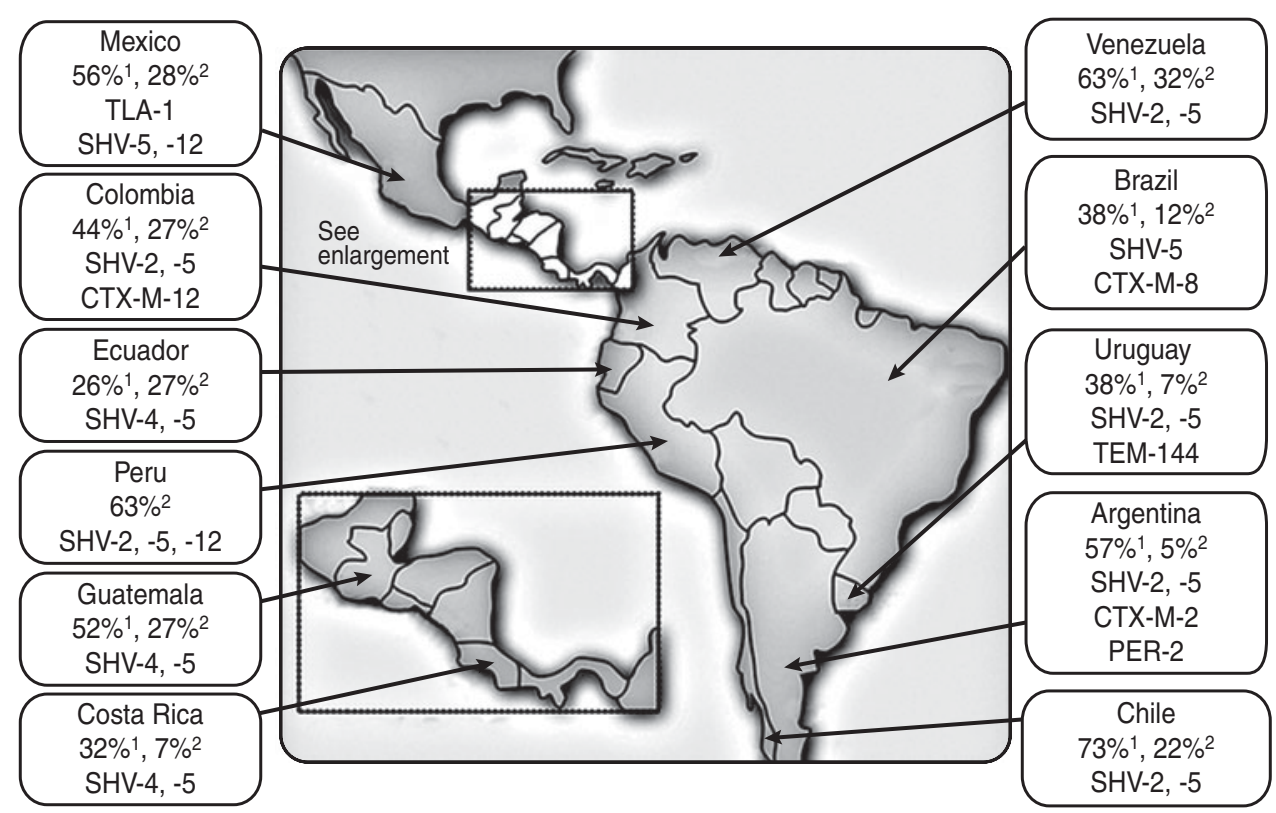

Fig. 1. Prevalence (and type) of extended spectrum $\beta$-lactamases harboured by $K$. pneumoniae ${ }^{1}$ and $E$. coli ${ }^{2}$ in Latin American clinical isolates during the late 1990s [32].

The CTX-M-type ESBLs have now reached endemic proportions in South America aided by the location of $b l a_{\mathrm{CTX}-\mathrm{M}}$ genes on plasmids and transposons, which engenders dissemination of CTX-Mproducing strains in different Enterobacteriaceae [30, 31, 33]. The spread of mobile genetic elements, mainly conjugative plasmids belonging to classic incompatibility groups, and the dispersion of specific clones have been responsible for the increase in ESBL-producing isolates and for the spread of many specific ESBLs, including CTX-M. ESBLs are often associated with co-resistance to fluoroquinolones, aminoglycosides, and trimethoprim/sulfamethoxazole, which may also have contributed to the current epidemiological ESBL scenario [33].

Also over the same time period, E. coli replaced Klebsiella spp. as the predominant species of ESBLproducing Enterobacteriaceae in the community, largely because of the ease of CTX-M mobilization [31]. Perhaps the high prevalence of communityacquired infection by $E$. coli-harbouring ESBLs was to be expected, given that these strains have been isolated from numerous sources such as domestic animals, food products, well water, sewage, and stool samples from healthy individuals [6, 34]. Importantly, there is evidence that community-associated E. coli ESBLs have infiltrated hospital settings [31, 34].

The most common community-acquired infection by Gram-negative bacteria is UTI, but clinicians are confronted with many different infection types including pneumonia and IAI $[19,20,35]$. E. coli, K. pneumoniae, and Proteus mirabilis are the most common organisms causing UTIs in ambulatory patients in Latin America [19-21, 35]. The aetiology of community-associated IAIs depends on the distribution of microflora at the anatomical site, although infections by enterobacteria (primarily E. coli and K. pneumoniae) tend to predominate [36].

\section{UTI}

The current rate of clinical failure associated with community-source uropathogens is unacceptably high, coincident with high levels of resistance to commonly used antimicrobials (Table 1, Fig. 2) [19, 25, 37-41]. Overall, data from four network studies [19, 25, 40, 41] as well as data from the Pan-American Health Organization (PAHO) surveillance system for 2009 and $2010[38,39]$, indicated that continent-wide resistance of $E$. coli to trimethoprim/sulfamethoxazole was high, resistance to quinolones was variable, and resistance to second-generation cephalosporins and gentamicin was routinely greater than $20 \%$. In contrast, rates of $E$. coli resistance to nitrofurantoin and fosfomycin were generally low.

Rates of ESBL production by community-source isolates have been reported only infrequently in the literature. In the SENTRY 2003 study, which collected 
Table 1. Percentage of drug-resistant community-acquired urinary tract E. coli isolates collected during five surveillance network studies

\begin{tabular}{|c|c|c|c|c|c|}
\hline \multirow[b]{3}{*}{ Antimicrobial } & \multicolumn{5}{|c|}{ Country (network), year (no. of isolates) } \\
\hline & \multirow{2}{*}{$\begin{array}{l}\text { Guatemala [40] } \\
2005-2010 \\
(n=1184)\end{array}$} & \multicolumn{2}{|c|}{$\begin{array}{l}\text { Ecuador } \\
\text { (REDNARBEC) [25] }\end{array}$} & \multirow{2}{*}{$\begin{array}{l}\text { Chilean } \\
\text { network [41] } \\
2009 \\
(n=36949)\end{array}$} & \multirow{2}{*}{$\begin{array}{l}\text { Argentina, Brazil, Chile, } \\
\text { Mexico, Venezuela } \\
(\text { SENTRY) [19] } \\
2003 \\
(n=403)\end{array}$} \\
\hline & & $\begin{array}{l}1999 \\
(n=1907)\end{array}$ & $\begin{array}{l}2007 \\
(n=6447)\end{array}$ & & \\
\hline Ampicillin & & 57 & 72 & 53.6 n.s. & $53 \cdot 6$ \\
\hline Ampicillin/sulbactam & $29 \cdot 3$ & 19 & 27 & & $23 \cdot 3$ \\
\hline Amoxicillin/clavulanate & & & & & $1 \cdot 2$ \\
\hline Piperacillin/tazobactam & $2 \cdot 9$ & & & $6 \cdot 4$ n.s. & $0 \cdot 2$ \\
\hline $\mathrm{C} 1 \mathrm{G}$ & & $25^{*}, 8 \dagger$ & $29 *, 9 \dagger$ & $31 \cdot 8 *$ n.s. & \\
\hline $\mathrm{C} 2 \mathrm{G}$ & & $3+$ & $9+$ & & $2 \cdot 2 \%$ \\
\hline $\mathrm{C} 3 \mathrm{G}$ & $2 \cdot 5 \S, 10 \cdot 8 \|$ & 19 & 39 & $5 \cdot 6 \|$ & $1 \cdot 5 \S, 1 \cdot 29$ \\
\hline $\mathrm{C} 4 \mathrm{G}$ & $8 \cdot 3 \#$ & & & & $1 \cdot 0 \#$ \\
\hline Cefoxitin & & & & & $1 \cdot 7$ \\
\hline Gentamicin & $21 \cdot 9$ & 3 & 18 & 6.9 n.s. & $8 \cdot 4$ \\
\hline Amikacin & $1 \cdot 4$ & $0 \cdot 5$ & $0 \cdot 9$ & $7 \cdot 5$ n.s. & 0 \\
\hline Ciprofloxacin & $46 \cdot 3$ & 18 & 41 & $19 \cdot 6$ n.s. & $21 \cdot 6$ \\
\hline $\begin{array}{l}\text { Trimethoprim/ } \\
\text { sulfamethoxazole }\end{array}$ & $58 \cdot 6$ & 50 & 57 & $29 \cdot 6$ n.s. & $40 \cdot 4$ \\
\hline Nitrofurantoin & $2 \cdot 5$ & 4 & 7 & 4 n.s. & $6 \cdot 9$ \\
\hline Ertapenem & & & & 0 n.s. & \\
\hline Imipenem & 0 & & & 0 n.s. & 0 \\
\hline Meropenem & 0 & & & 0 n.s. & \\
\hline Fosfomycin & & 1 & 2 & & \\
\hline
\end{tabular}

REDNARBEC, Red Nacional de Resistencia Bacteriana de Ecuador; C1G, First-generation cephalosporins; C2G, second-generation cephalosporins; $\mathrm{C} 3 \mathrm{G}$, third-generation cephalosporins; $\mathrm{C} 4 \mathrm{G}$, fourth-generation cephalosporins; n.s., non-susceptible.

* Cefalotin; $\dagger$ cefazolin; $\$$ cefuroxime; $\S$ ceftazidime; $\|$ cefotaxime; $\uparrow$ ceftriaxone; \# cefepime.

data from Argentina, Brazil, Chile, Mexico and Venezuela, rates of ESBL production were $1.7 \%$, $16 \cdot 3 \%$, and $5 \cdot 1 \%$ in urinary isolates of $E$. coli, Klebsiella spp., and P. mirabilis, respectively [19]. The rate of ESBL production in E. coli isolates was $16 \%$ in outpatients in Guatemala during the period 2005-2010 [40].

\section{Central America}

The PAHO 2010 data for isolates of community origin reported high resistance rates of $E$. coli to trimethoprim/sulfamethoxazole and ciprofloxacin throughout Central America (Fig. 2), as well as low resistance rates of $E$. coli to nitrofurantoin $(\leqslant 17 \%)$. Resistance of $E$. coli to cefuroxime ranged from $10 \%$ (in Panama) to $42 \%$ (females in Honduras); resistance to gentamicin ranged from $4 \%$ (in Guatemala) to $28 \%$ (males in Honduras) [39]. In the 4 years since PAHO
2006 surveillance data were collected, PAHO 2010 surveillance data indicated that resistance had edged higher to most antimicrobial drug classes in those countries with available data (i.e. El Salvador, Honduras, Nicaragua).

Resistance rates of $E$. coli isolates collected in a surveillance study in Guatemala in 2005-2010 are shown in Table 1. In Mexican data from the SENTRY 2003 study, antimicrobial susceptibility rates of urinary E. coli isolates were as follows: ampicillin, $22 \%$; nalidixic acid, 25\%; levofloxacin, 28\%; gatifloxacin, 31\%; trimethoprim/sulfamethoxazole, 39\%; amoxicillin/ clavulanate, $56 \%$; cefuroxime, $64 \%$; and nitrofurantoin, $81 \%$ [19]. Although the nitrofurantoin susceptibility data in SENTRY 2003 data for Mexico were encouraging, increased utilization of this antimicrobial agent as a recommended first-line agent appeared to induce resistance in a study of 304 patients with suspicion of UTI at the university hospital and primary health 
(a)

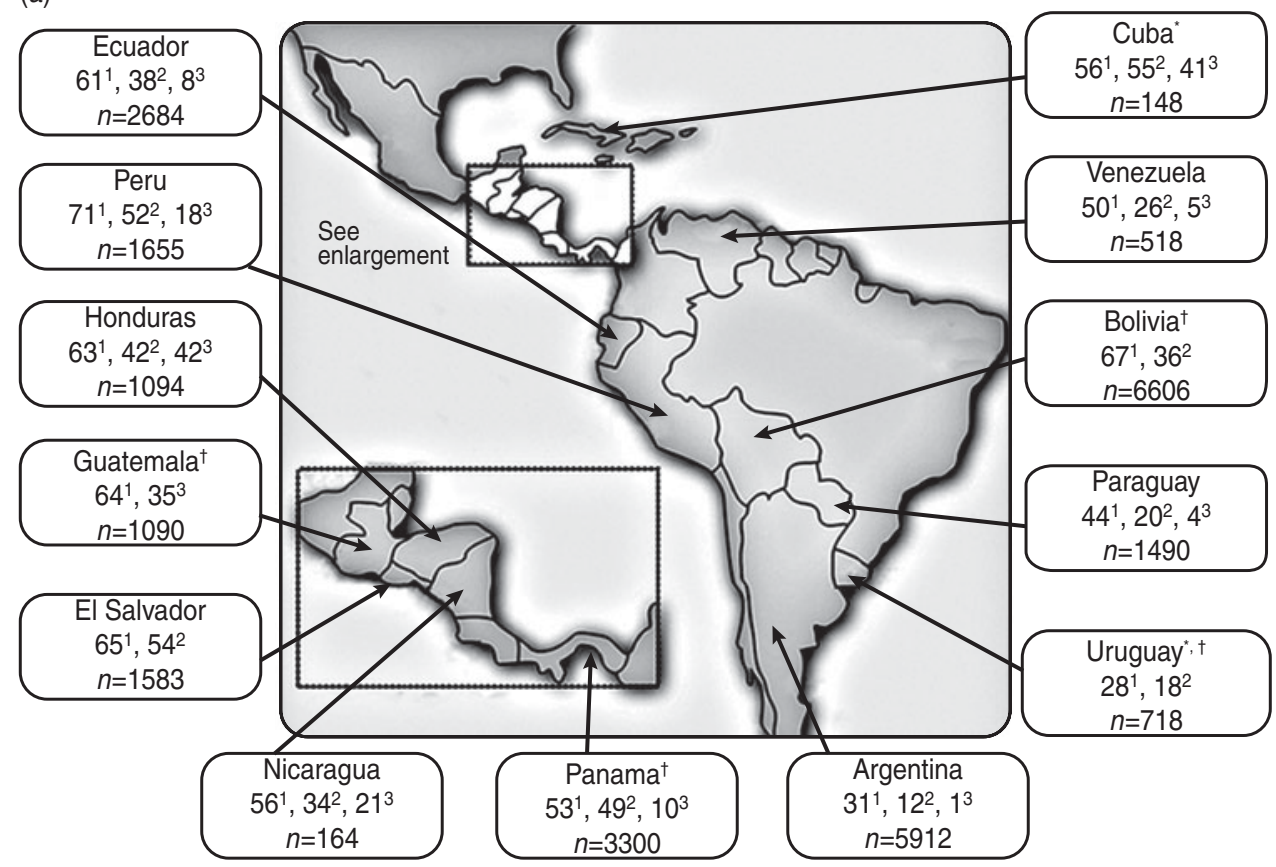

(b)

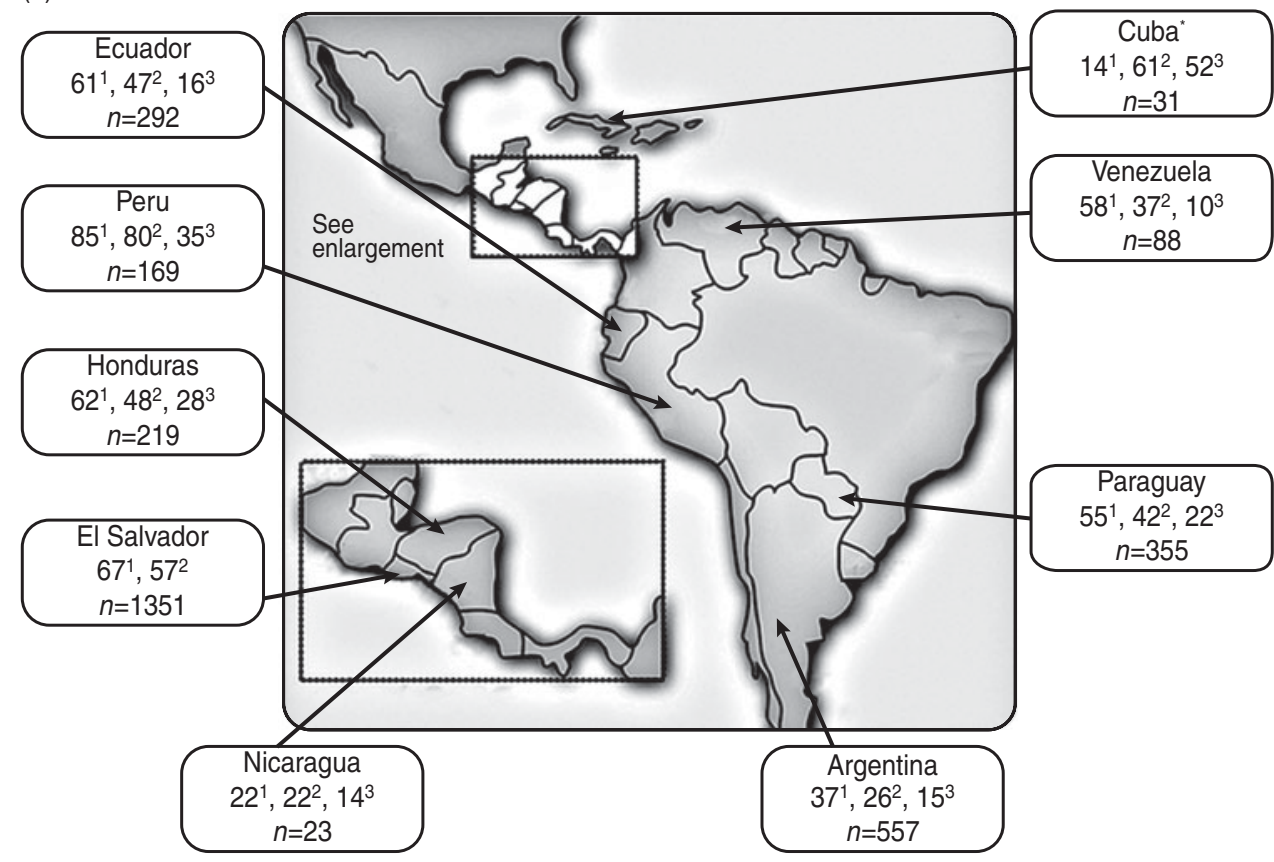

Fig. 2. Percentage (no. of isolates) of urinary tract $E$. coli isolates collected from $(a)$ women and $(b)$ men in Latin America (2010 PAHO report) that were resistant to trimethoprim/sulfamethoxazole ${ }^{1}$, ciprofloxacin ${ }^{2}$, and the second-generation cephalosporin cefuroxime ${ }^{3}$ [39]. * Data from the 2009 PAHO report [38] is given for this country because 2010 data were not reported; $\dagger$ all adults (women and men).

centres of León, Nicaragua [20]. In the 5 years after the introduction of the therapeutic guidelines, resistance of E. coli against nitrofurantoin increased from $0 \%$ to $7 \%$ [20]. In this study [20], high resistance rates of E. coli were also observed in 2008 with ampicillin $(61 \%)$, cefalotin $(46 \%)$, trimethoprim/sulfamethoxazole
$(39 \%)$, ciprofloxacin $(32 \%)$, gentamicin $(25 \%)$, and ceftriaxone $(20 \%)$. Thirteen $(30 \%)$ of $44 \mathrm{E}$. coli strains were suspected of producing ESBLs, with resistance rates in ESBL-producing $E$. coli significantly higher to ampicillin ( $85 \%$ vs. $52 \%)$, amoxicillin/clavulanate $(46 \%$ vs. $6 \%)$, cefalotin $(85 \%$ vs. $29 \%)$, ceftriaxone 
(69\% vs. $0 \%)$, and ciprofloxacin $(62 \%$ vs. $19 \%)$ compared to pathogens that did not produce ESBLs [20].

\section{South America}

The PAHO 2010 data for isolates of community origin revealed that resistance of E. coli to trimethoprim/sulfamethoxazole ranged from $31 \%$ to $85 \%$ across South American countries, and resistance to ciprofloxacin ranged from $12 \%$ to $80 \%$, with the lowest and highest resistance rates for both antimicrobial agents occurring in Argentina and Peru (Fig. 2) [39]. E. coli resistance to nitrofurantoin was low (i.e. $<20 \%$ ) except in Argentinian males (31\%). In contrast, resistance to gentamicin was erratic, ranging from $4 \%$ in Uruguay to $29 \%$ in Peruvian males [39].

Eight-year temporal data reported in the Red Nacional de Resistencia Bacteriana de Ecuador (REDNARBEC) study are shown in Table 1. Of the South American countries that participated in SENTRY 2003 (Argentina, Brazil, Chile, Venezuela; Table 1), susceptibility rates were generally comparable across countries [19]. The greatest difference was for the fluoroquinolones, with susceptibility to levofloxacin ranging from $72 \%$ in Venezuela to $91 \%$ in Brazil [19].

Results of a large Brazilian study conducted in Curitiba found that few suitable empirical treatment options for community-source UTIs were available for women aged $>60$ years and males of any age [37]. Of 9798 consecutive, non-duplicate, community-source urine isolates from ambulatory patients aged $>13$ years during 2009 , E. coli $(66 \%)$ was by far the most prevalent Gram-negative pathogen followed by Klebsiella spp. (6\%), P. mirabilis (4\%), and Enterobacter spp. (3\%) [37]. Susceptibility of E. coli varied widely by drug class, being very low for ampicillin and trimethoprim/ sulfamethoxazole ( $56 \%$ and $66 \%$, respectively), suboptimal for fluoroquinolones (82\%), and acceptable for gentamicin, ceftriaxone/cefotaxime and nitrofurantoin (93\%, 97\% and 96\%, respectively) [37]. Importantly, the susceptibility rates of $E$. coli urinary isolates were $3-4 \%$ higher for fluoroquinolones and gentamicin, and at least $30 \%$ higher for nitrofurantoin and extendedspectrum cephalosporins, compared to susceptibility rates for other pathogens causing community-source urinary infections [37].

\section{IAI}

The Study for Monitoring Antimicrobial Resistance Trends (SMART) monitors the activity of key anti- microbial drug classes against Gram-negative bacteria isolated from IAIs to ensure that the current susceptibility patterns of these organisms are well understood and reported effectively [36]. The number of participating sites in Latin America increased from six in 2002 to 13 in 2007, with 740 isolates sent for analysis on average each year [36]. In 2008, there were 23 centres in ten Latin American countries (Argentina, Brazil, Chile, Colombia, Dominican Republic, Guatemala, Mexico, Panama, Peru, Venezuela) that participated in SMART, with each centre collecting up to 100 consecutive non-duplicate clinical isolates from patients with IAI [42]. Of 1003 Gram-negative bacilli isolates collected from IAIs, E. coli $(50 \%), K$. pneumoniae $(15 \%)$, and Enterobacter cloacae $(7 \%)$ were the most commonly isolated pathogens $(4 \%$ of isolates were P. mirabilis) [42]. More than one quarter of E. coli $(27 \%)$ and more than one third of $K$. pneumoniae $(38 \%)$ isolates were ESBL-positive. The prevalence of ESBL-producing E. coli and Klebsiella spp. associated with community-acquired infections in particular was $29 \%$ [42].

In the SMART study, susceptibilities of ESBLproducing strains to antibacterial agents commonly used in the community such as third-generation cephalosporins, fluoroquinolones, and ampicillin/sulbactam were low (Table 2) [42]. Six-year temporal data from SMART indicate that the percentages of susceptible ESBL-positive $E$. coli and $K$. pneumoniae isolates to ciprofloxacin and cefepime are declining, whereas susceptibilities to amikacin are steady (Fig. 3) [42].

The phenotypes of $E$. coli isolated from 19 SMART investigator sites in 11 Latin American countries during 2008-2009 were determined [43]. Of the 1366 isolates, $323(24 \%)$ produced ESBLs, which is an increase from $12 \%$ in 2004 and $22 \%$ in $2005-2007$. The proportion of isolates that were ESBL-producing varied widely in Latin American countries (Fig. 4) [43]. ESBL production had a major deleterious effect on activity against ampicillin/sulbactam, cephalosporins, and fluoroquinolones, as well as on the activity of amikacin, perhaps indicating a co-resistance phenomenon (Table 3) [43]. It has been postulated that fluoroquinolone resistance is a harbinger of broader antimicrobial resistance, including ESBL selection [44].

\section{RISK FACTORS}

Early recognition of patients at a heightened risk for infection with multidrug-resistant bacteria 


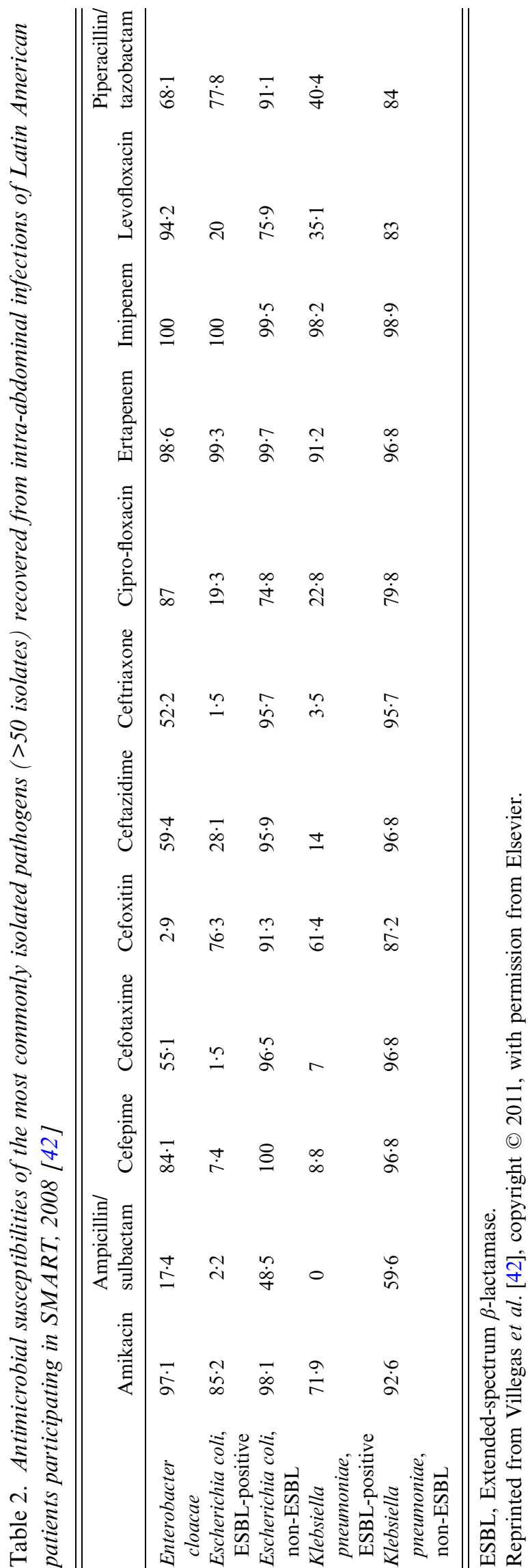

is necessary to provide appropriate empirical treatment and institute measures that control or limit disease transmission. This observation is especially true in cases of IAI since ampicillin/sulbactam, thirdgeneration cephalosporins, and fluoroquinolones have a limited role as first-line treatments for IAIs. Risk factors for infection with ESBL-producing Enterobacteriaceae have been identified in several studies of hospitalized patients [45-49]. Risk factors identified in multivariate analyses in these studies include previous antibiotic use (specifically, use of quinolones [47], cephalosporins [45], oxyiminocephalosporins [46], piperacillin/tazobactam [45], or $\beta$-lactams with an oxyimino group [48]), recurrent infections [47], haemodialysis [49], urinary catheterization [46], artificial nutrition [47], and residence in a nursing home [49].

The relevance of risk factors in the hospital setting to the community setting is unclear. According to Pitout \& Laupland [26], repeated episodes of UTI and underlying renal pathology, previous use of antibiotics including cephalosporins and quinolones, previous hospitalization, nursing home residence, older age, presence of diabetes mellitus, and underlying liver pathology are risk factors for community-onset infections caused by ESBL-producing bacteria [26]. A meta-analysis of epidemiological studies of infection caused by ESBL-producing Enterobacteriaceae in non-hospitalized patients from six centres in Europe, Asia, and North America revealed that recent use of any antibiotic, residence in a long-term care facility, recent hospitalization, age $\geqslant 65$ years, and male sex were statistically significant risk factors for infection by an ESBL-producing organism [34]. However, it can be argued that emergence of ESBL-producing bacteria, primarily CTX-M-type $\beta$-lactamases, confounds infection control strategies based on traditional risk factors, since one third of ESBL-producing isolates in the meta-analysis were obtained from patients with no recent healthcare contact [34]. Multivariate analysis of a nested case-control study of 787 consecutive patients presenting with febrile UTI in 2004-2009 in The Netherlands revealed recent hospitalization, presence of a urinary catheter, and fluoroquinolone use in the past 6 months to be independent risk factors for infection due to fluoroquinolone-resistant E. coli [44]. In summary, risk factors for infection by ESBL-producing bacteria in the community setting are similar to those in the hospital setting and are fairly consistent across studies. 

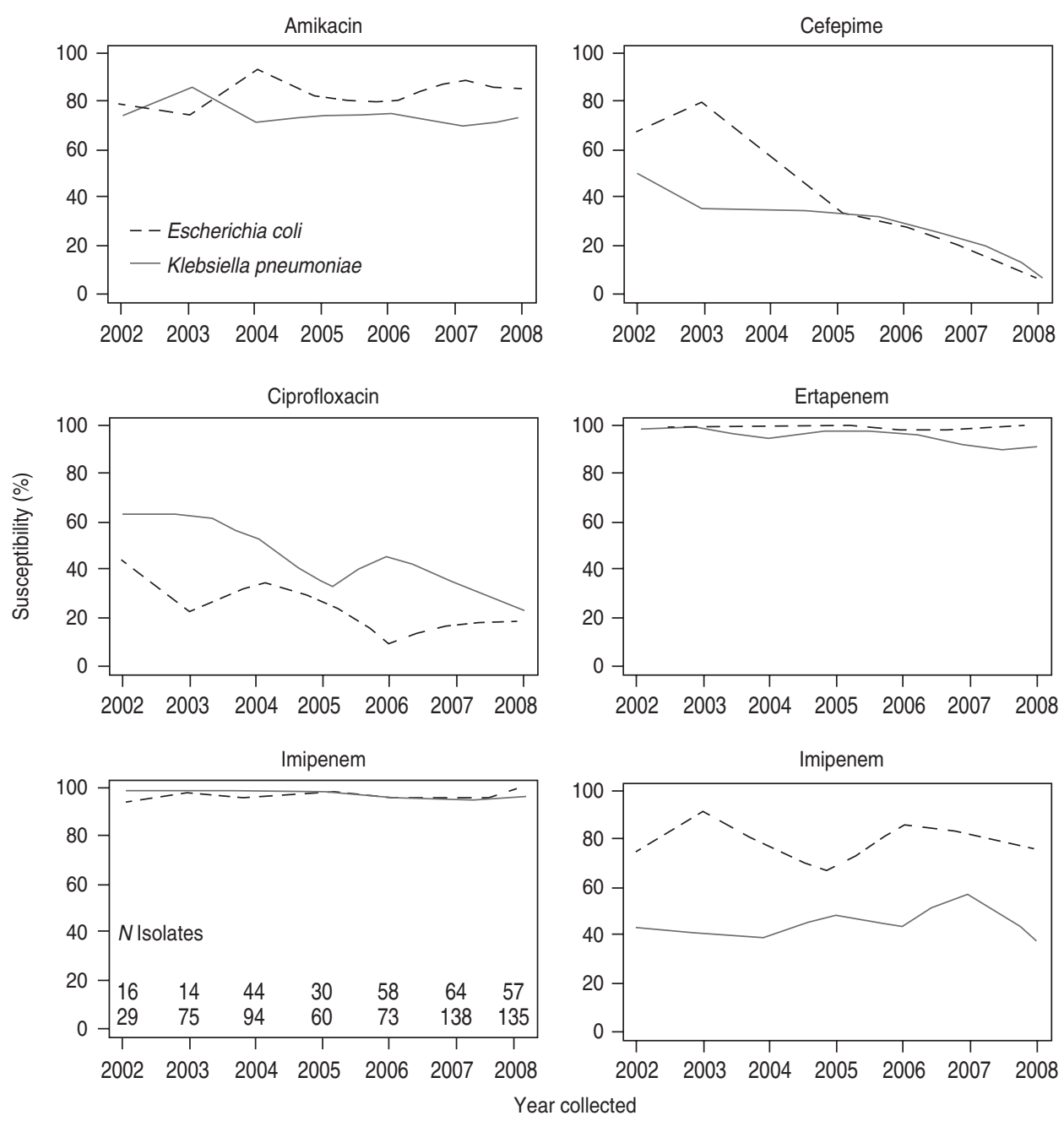

Fig. 3. Antimicrobial susceptibilities of ESBL-producing E. coli and $K$. pneumoniae intra-abdominal isolates in Latin America (2002-2008). Susceptibilities are based on in vitro minimum inhibitory concentration data. (Reprinted from Villegas et al. [42], copyright $\odot$ 2011, with permission from Elsevier.)

\section{SURVEILLANCE AND DETECTION}

With the advent of widespread dispersion of ESBLproducing Enterobacteriaceae in communities of Latin America, empirical prescribing of antimicrobial agents according to general principles can no longer ensure effective treatment. Rather, local susceptibility patterns attained within each institution and maintenance of longitudinal surveillance programmes are needed to inform treatment decisions. Surveillance also aids patient diagnosis and facilitates infection control strategies. Despite an investment to survey bacterial resistance in Latin America through PAHO, SENTRY, SMART and other programmes, further efforts are required to more fully integrate these resources in a practical way so that real-time data regarding antimicrobial susceptibility and genotypic patterns are available to healthcare professionals [28].
Aside from these overarching logistical issues, there are also major limitations in our capability to detect ESBL-producing Enterobacteriaceae. These strains confound traditional surveillance strategies because ESBL production cannot be inferred from the antimicrobial resistance profile alone. Hence, the emergence, maintenance, and dissemination of ESBLs must be characterized and closely monitored by implementation of integrated phenotypic and genotypic testing strategies. To give some scale to the magnitude of the task ahead, the number of CTX-M variants alone numbered 138 as of March 2013 [50].

\section{Phenotypic methods}

Most clinical microbiology laboratories in Latin America conduct susceptibility testing as it is easy, automated, inexpensive, and accessible; however, 
Table 3. Antimicrobial susceptibilities of 323 ESBL-positive intra-abdominal E. coli isolates tested in SMART 2008-2009 based on Clinical and Laboratory Standards Institute breakpoints [43]

\begin{tabular}{|c|c|c|c|c|}
\hline Antimicrobial & $\operatorname{MIC}_{90}(\mu \mathrm{g} / \mathrm{ml})$ & Susceptible (\%) & $\begin{array}{l}\text { Intermediate } \\
\text { susceptible }(\%)\end{array}$ & Resistant (\%) \\
\hline Ampicillin/sulbactam & $>16(>16)$ & $6 \cdot 8(42 \cdot 8)$ & $23 \cdot 5(23 \cdot 1)$ & $69 \cdot 7(34 \cdot 1)$ \\
\hline Piperacillin/tazobactam & $64(16)$ & $80 \cdot 5(91 \cdot 7)$ & $12 \cdot 7(3 \cdot 2)$ & $6 \cdot 8(5 \cdot 1)$ \\
\hline Cefepime & $>32(\leqslant 0 \cdot 5)$ & $11 \cdot 8(100)$ & $2 \cdot 2(0)$ & $86 \cdot 0(0)$ \\
\hline Cefotaxime & $>128(\leqslant 0 \cdot 5)$ & $0 \cdot 9(94 \cdot 3)$ & $0 \cdot 9(1 \cdot 0)$ & $98 \cdot 2(4 \cdot 7)$ \\
\hline Cefoxitin & $16(8)$ & $79 \cdot 6(92 \cdot 2)$ & $14 \cdot 5(1 \cdot 7)$ & $5 \cdot 9(6 \cdot 1)$ \\
\hline Ceftazidime & $>128(\leqslant 0 \cdot 5)$ & $21 \cdot 4(95 \cdot 1)$ & $4 \cdot 0(1 \cdot 1)$ & $74 \cdot 6(3 \cdot 8)$ \\
\hline Ceftriaxone & $>32(\leqslant 1)$ & $0 \cdot 9(94 \cdot 1)$ & $0.6(0 \cdot 7)$ & $98 \cdot 5(5 \cdot 2)$ \\
\hline Amikacin & $32(8)$ & $88 \cdot 8(98 \cdot 4)$ & $5 \cdot 6(1 \cdot 2)$ & $5 \cdot 6(0 \cdot 5)$ \\
\hline Ciprofloxacin & $>2(>2)$ & $16 \cdot 4(69 \cdot 2)$ & $0 \cdot 0(0 \cdot 6)$ & $83 \cdot 6(30 \cdot 2)$ \\
\hline Levofloxacin & $>4(>4)$ & $17 \cdot 3(70 \cdot 4)$ & $1 \cdot 6(2 \cdot 1)$ & $81 \cdot 1(27 \cdot 5)$ \\
\hline Ertapenem & $0 \cdot 25(\leqslant 0.03)$ & $95 \cdot 1(99 \cdot 1)$ & $4 \cdot 0(0 \cdot 8)$ & $0 \cdot 9(0 \cdot 1)$ \\
\hline Imipenem & $0 \cdot 25(0 \cdot 12)$ & $99 \cdot 4(99 \cdot 3)$ & $0 \cdot 3(0 \cdot 4)$ & $0 \cdot 3(0 \cdot 3)$ \\
\hline
\end{tabular}

ESBL, Extended-spectrum $\beta$-lactamase.

Values in parentheses are the corresponding data pertaining to the 1043 ESBL-negative isolates tested in SMART $2008-2009$.

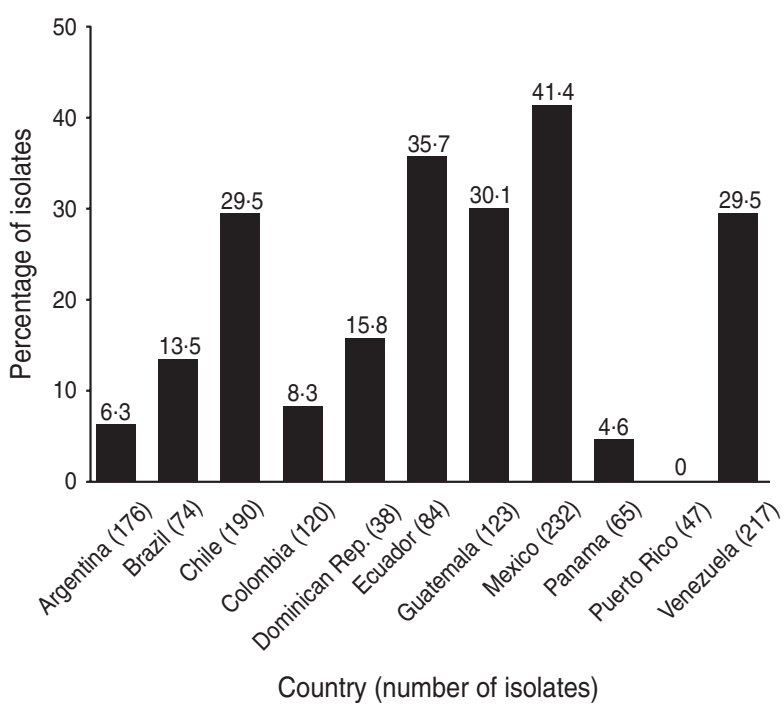

Fig. 4. Proportion of E. coli isolates from intra-abdominal infections in Latin America that were extended-spectrum $\beta$-lactamase positive (SMART, 2008-2009) [43].

phenotypic methods cannot provide information on the type of ESBL produced. The value of nonmolecular phenotypic methods is premised on the fact that most ESBLs hydrolyse third-generation cephalosporins and are inhibited by clavulanate [26]. Numerous methods have been developed to detect or confirm ESBL production by Enterobacteriaceae. Automated systems screen isolates and confirm ESBL production based on minimum inhibitory concentrations of cefotaxime and ceftazidime with and without clavulanic acid.
However, the most appropriate choice of extendedspectrum cephalosporins and type of confirmatory test that provides the greatest assay sensitivity have yet to be determined. For instance, in early testing, the E-test ESBL screen test with ceftazidime or cefepime as substrate, and use of Oxoid combination discs with cefotaxime and ceftazidime, were useful for demonstrating the presence of Enterobacteriaceae potentially producing ESBLs but were also associated with clinically significant false-susceptible and false-resistant results [51, 52]. Current guidance from the Clinical and Laboratory Standards Institute (CLSI) describes laboratory detection of ESBL produced by E. coli, P. mirabilis, and Klebsiella spp., but not for species with inducible AmpC $\beta$-lactamases (such as Enterobacter spp.) [53]. Specifically, for ESBL detection in Enterobacteriaceae, CLSI recommends initial screening with $8 \mu \mathrm{g} / \mathrm{ml}$ of cefpodoxime; $1 \mu \mathrm{g} / \mathrm{ml}$ each of cefotaxime, ceftazidime, ceftriaxone, or aztreonam; followed by confirmatory tests (including the E-test ESBL strips) with both cefotaxime and ceftazidime in combination with clavulanate at a concentration of $4 \mu \mathrm{g} / \mathrm{ml}$ [54]. As of 2007, high sensitivities of up to $94 \%$ and specificity of $98 \%$ for detecting ESBLs in E. coli, Klebsiella spp, and Proteus spp. were expected if these techniques were adhered to [55].

Recent emergence of plasmidic AmpC $\beta$-lactamases harboured by E. coli and $K$. pneumoniae may result in changes to CLSI recommendations. The phenotypic detection of ESBLs in bacteria other than E. coli, Klebsiella spp., and Proteus spp. will remain problematical because of the possible association 
of resistance mechanisms, presence of more than one $\beta$-lactamase [especially carbapenemases of the $K$. pneumoniae carbapenemase (KPC) type], and reduced permeability.

\section{Genotypic methods}

Genotypic methods use molecular biology techniques to detect the gene responsible for ESBL production, with the aim of distinguishing between resistance genes, genetic elements, and strains. The primary technology used is polymerase chain reaction (PCR) amplification of $b l a_{\mathrm{TEM}}, b l a_{\mathrm{SHV}}$, and bla $\mathrm{CTX}_{\mathrm{CT}-\mathrm{M}}$ genes with oligonucleotide primers followed by sequencing to discriminate between non-ESBL parent enzymes and their ESBL variants [26]. ESBLs have also been characterized by PCR restriction fragment-length polymorphism and single-strand conformational polymorphism, and by restriction site insertion PCR, realtime PCR, and ligase chain reaction [26]. A limitation of PCR is that it detects ESBL genes but does not inform on ESBL production. In addition, few clinical microbiology laboratories in Latin America are equipped to perform recombinant DNA techniques, which are complicated, time-consuming, and expensive in part because of the presence of multiple copies of ESBLs in any given clinical isolate.

Whole-genome sequencing and multilocus sequence typing has tremendous utility, as it enables microbiologists to characterize the population biology of a species, and thus track the evolution and spread of clones. Currently, these techniques are confined to reference laboratories and have little influence on local and regional infection control programmes.

\section{Clonality in Latin America}

The CTX-M family includes a heterogeneous group of ESBLs divided into five groups based on primary structure (CTX-M-1, CTX-M-2, CTX-M-8, CTXM-9, CTX-M-25) [26]. Although CTX-Ms are produced by a wide variety of Enterobacteriaceae strains, CTX-M globalization is associated with a few clones of $E$. coli and $K$. pneumoniae, which underscores the selective advantage of expressing these enzymes. Most clinical isolates are not clonally related, but clonal outbreaks have been described in several countries $[26,56]$. It has been estimated that at least $10-20 \%$ of all UTIs are caused by clonally related E. coli, which are often co-resistant to aminoglycosides and trimethoprim/sulfamethoxazole [33, 56].
Detecting successful strains or epidemic clones from large volumes of isolates with the same phenotype is challenging [28].

E. coli belonging to phylogroup B2 [sequence type 131 (ST131)] and phylogroup D (ST405) are the most infamous community-associated, high-risk clones because of their rapid globalization coupled with high virulence and multidrug-resistant IncFII plasmids [15, 57]. E. coli ST131 has been implicated in severe community-acquired infections, including septicaemia [26, 58]. In Latin America, E. coli ST131 was initially detected in the Colombian and Brazilian hospital setting in 2008 [59, 60] and subsequently in the Colombian community setting in 2010 (along with clone ST405) and possibly in 2011 [61, 62]. The ease with which $E$. coli ST131 diffuses through communities via infected or colonized family members, wildlife, foodstuffs, and companions represents a major public health concern [15].

The composition of a different high-risk clone of E. coli causing community-acquired UTIs in Rio de Janeiro, Brazil, between 2005 and 2006 was determined by a cross-sectional study of 344 women seeking care in one public walk-in clinic [63]. More than half (54\%) of the women had a documented UTI, of which $63 \%$ were caused by E. coli. Of the $50 \%$ of E. coli isolates resistant to ampicillin and trimethoprim/sulfamethoxazole, most $(81 \%)$ belonged to 19 enterobacterial repetitive intergenic consensus (ERIC2) clonal groups. All isolates in the largest clonal group ( $n=15$ isolates) belonged to multilocus sequence typing group ST69 and phylogenetic group D, and had $89 \%$ similarity to a clonal group $\mathrm{A}(\mathrm{CgA})$ reference strain from the USA [63]. These data indicate that uropathogenic E. coli $\mathrm{CgA}$ strains have mobilized from North America and Europe to Latin America, where they have the potential to cause multidrug-resistant outbreaks.

Brazil has a particularly high diversity of CTX-M enzymes harboured by clinical isolates of $K$. pneumoniae and $E$. coli. In a community and hospital setting in Rio de Janeiro (2000-2001), analysis of the epidemiological features of $41 \mathrm{E}$. coli isolates resistant to third-generation cephalosporins and/or non- $\beta$-lactam antibiotics revealed a high prevalence of CTX-M-2 production [64]. CTX-M-9 and CTX-M-59 (a variant of CTX-M-2) were also identified. Of note, the CTX-M-producing E. coli in this study belonged to different phylogroups/sequence types that were associated with IncA/C plasmids implicated in the facilitation of CTX-M globalization and evolution [64]. 
The first report of a Citrobacter freundii-producing CTX-M-14 isolated from a woman in Venezuela with a recurrent UTI highlights the problem of plasmid-mediated dissemination of these $\beta$-lactamases into other bacterial populations [65].

\section{PROPOSALS FOR LATIN AMERICA}

When reviewing the data, we recognize that betterdesigned studies are urgently required to more accurately quantify the epidemiology of Gram-negative community-associated infections in Latin America. Rather than use local criteria, these studies should report on a standardized definition for communityassociated UTIs and IAIs and use approved methods for phenotypic and genotypic testing. Furthermore, local and national susceptibility data are required on a far greater number of isolates before treatment algorithms can be developed.

Even taking into consideration the gaps in our knowledge regarding the epidemiology of community-associated UTIs and IAIs by Gram-negative bacteria, the weight of evidence suggests that primarycare physicians in Latin America should consider the potential for involvement of multidrug-resistant bacteria when managing cases of UTI and IAI. Published guidelines (including recent guidelines of the Infectious Diseases Society of America for treatment of acute uncomplicated cystitis [66]) can be consulted for general guidance, but local conditions, including availability of specific agents, will influence treatment. (Fosfomycin, for example, may be used in the correct formulation for treatment of UTI in women.) Severity of infection as well as risk factors for infection by multidrug-resistant Enterobacteriaceae can be used to inform treatment decisions. If available, knowledge of local antimicrobial susceptibility data should be used as a reference guide. However, we recommend that urine cultures be collected for all recurrent or relapsing UTIs, complicated UTIs, and UTI cases presenting to the emergency room. This assertion is supported by the high levels of resistance of urinary E. coli isolates to trimethoprim/sulfamethoxazole and quinolones across the continent, rendering prescribing decisions difficult without supportive microbiological data [19, 39]. Urine cultures are inexpensive to perform and are readily available in most hospitals of Latin America. Furthermore, obtaining cultures is necessary to implement antimicrobial stewardship programmes, in which de-escalation is a very important method to decrease selective pressure on broad-spectrum antibiotics. Primary-care physicians, including gynaecologists, should also be educated that asymptomatic patients who receive a positive test for bacteria growth in urine should not be treated with antibiotics, as the positive test usually indicates colonization and not infection.

\section{CONCLUSION}

Overall, data describing the microbiology of community-associated UTIs and IAIs caused by Gram-negative bacteria in Latin America over the last 10 years indicate high rates of continent-wide resistance to trimethoprim/sulfamethoxazole, quinolones, second-generation cephalosporins, and gentamicin, and low levels of resistance to third- and fourth-generation cephalosporins, nitrofurantoin, and fosfomycin. We report $E$. coli resistance rates to quinolones routinely greater than $20 \%$ (and up to $80 \%$ ), which is higher than the 2009 national average in the USA $(19 \cdot 5 \%)$ [67]. Widespread use of quinolones in humans and in animal husbandry in Latin America may account for this difference. The concern around the endemicity of quinolone-resistant $E$. coli in Latin America is the association with plasmidmediated quinolone resistance, which accelerates the rate at which other Enterobacteriaceae develop fluoroquinolone resistance. The extremely high rate of E. coli resistance to trimethoprim/sulfamethoxazole probably reflects widespread use and misuse of this low-cost antimicrobial agent for treatment of community-associated UTI in the region. Findings from the publications assessed in this review tentatively indicate that ESBL rates in E. coli-causing IAIs are variable but increasing over time, although not enough data are available to confirm the seriousness of this problem [43]. The rate of ESBLs harboured by urinary isolates of $E$. coli requires further study, given the ease of CTX-M mobilization, high potential for clonal outbreaks, and increase in reports of UTIs by ceftriaxone-resistant $E$. coli.

Given the high resistance rates in Enterobacteriaceae causing community-acquired UTIs and IAIs and lack of therapeutic options, we recommend that antimicrobial prescribing be guided by considering infection severity, established patient risk factors for multidrug-resistant infections, acquaintance with local antimicrobial susceptibility data, and culture collection. 


\section{APPENDIX: Latin America Working Group on Bacterial Resistance}

Carlos Alvarez (Hospital Universitario San Ignacio and Pontificia Universidad Javeriana, Bogotá, Colombia), Luis Bavestrello (Clinica Reñaca, Viña Del Mar, Chile), Eitan Berezin (Santa Casa de São Paulo School of Medicine, Brazil), Eduardo Gotuzzo (Universidad Peruana Cayetano Heredia, Lima, Perú), Manuel Guzmán-Blanco (Hospital Privado Centro Médico de Caracas, Venezuela), Jaime A. Labarca (Pontificia Universidad Católica de Chile, Santiago, Chile), Carlos M. Luna (Hospital de Clínicas José de San Martin Hospital, Universidad de Buenos Aires, Argentina), Carlos Mejía (Hospital Roosevelt, Guatemala City, Guatemala), Simone Nouer (Hospital Universitario Clementino Fraga Filho, Rio de Janeiro, Brazil), Eduardo Rodríguez-Noriega (Hospital Civil de Guadalajara Fray Antonio Alcalde, Guadalajara, Mexico), Mauro José Costa Salles (Hospital Irmandade da Santa Casa de Misericórdia de São Paulo, Brazil), Carlos Seas (Universidad Peruana Cayetano Heredia, Lima, Perú), Fortino Solórzano Santos (Hospital de Pediatría Centro Médico Nacional Siglo XXI, Mexico City, Mexico), Maria Virginia Villegas [International Center for Medical Research and Training (CIDEIM), Cali, Colombia], Jeannete Zurita (Hospital Vozandes and Pontificia Universidad Católica del Ecuador, Quito, Ecuador).

\section{ACKNOWLEDGEMENTS}

This publication was funded by Pfizer Inc. Medical writing support was provided by Malcolm Darkes and Lisa Baker of UBC Scientific Solutions and was funded by Pfizer Inc.

\section{DECLARATION OF INTEREST}

Dr Mauro José Costa Salles is an advisory board member for Pfizer and Novartis; a consultant or speaker for Pfizer, Merck Sharp \& Dohme, Novartis and United Medicals; and a recipient of research grants from Pfizer and Novartis. Dr Jeannete Zurita is an Advisory Board member and consultant for Pfizer, and received research grants from Wyeth (now part of Pfizer) and from Merck. Dr Carlos Mejía participated in advisory groups for HIV treatment in association with Abbott, GlaxoSmithKline, and Stendhal. He participated in the SMART study, which was financially supported by Merck, and is a member of an advisory group for Pfizer. Dr Maria Virginia Villegas has received consulting fees and research grants from Merck Sharp \& Dohme, Pfizer SA, Janssen-Cilag SA, Novartis, and AstraZeneca Colombia SA.

\section{REFERENCES}

1. Fornasini M, et al. Trimethoprim-resistant Escherichia coli in households of children attending day care centers. Journal of Infectious Diseases 1992; 166: 326-330.

2. Reves RR, et al. Risk factors for fecal colonization with trimethoprim-resistant and multiresistant Escherichia coli among children in day-care centers in Houston, Texas. Antimicrobial Agents and Chemotherapy 1990; 34: $1429-1434$.

3. McKeon DM, Calabrese JP, Bissonnette GK. Antibiotic resistant Gram-negative bacteria in rural groundwater supplies. Water Research 1995; 29: 1902-1908.

4. Woerther PL, et al. Emergence and dissemination of extended-spectrum beta-lactamase-producing Escherichia coli in the community: lessons from the study of a remote and controlled population. Journal of Infectious Diseases 2010; 202: 515-523.

5. Hawkey PM, Jones AM. The changing epidemiology of resistance. Journal of Antimicrobial Chemotherapy 2009; 64 (Suppl. 1): 3-10.

6. Amaya E, et al. Antibiotic resistance patterns of Escherichia coli isolates from different aquatic environmental sources in León, Nicaragua. Clinical Microbiology and Infection 2012; 18: E347-354.

7. Amábile-Cuevas C. Antibiotic resistance in Mexico: a brief overview of the current status and its causes. Journal of Infection in Developing Countries 2010; 4: 126-131.

8. Reinert RR, et al. Antimicrobial susceptibility among organisms from the Asia/Pacific Rim, Europe and Latin and North America collected as part of TEST and the in vitro activity of tigecycline. Journal of Antimicrobial Chemotherapy 2007; 60: 1018-1029.

9. Eisenberg $\mathbf{J N}$, et al. In-roads to the spread of antibiotic resistance: regional patterns of microbial transmission in northern coastal Ecuador. Journal of the Royal Society Interface 2012; 9: 1029-1039.

10. Dierikx CM, et al. Occurrence and characteristics of extended-spectrum-beta-lactamase- and AmpCproducing clinical isolates derived from companion animals and horses. Journal of Antimicrobial Chemotherapy 2012; 67: 1368-1374.

11. Carattoli A, et al. Extended-spectrum beta-lactamases in Escherichia coli isolated from dogs and cats in Rome, Italy, from 2001 to 2003. Antimicrobial Agents and Chemotherapy 2005; 49: 833-835.

12. Dierikx C, et al. Increased detection of extended spectrum beta-lactamase producing Salmonella enterica and Escherichia coli isolates from poultry. Veterinary Microbiology 2010; 145: 273-278.

13. Hunter PA, et al. Antimicrobial-resistant pathogens in animals and man: prescribing, practices and policies. 
Journal of Antimicrobial Chemotherapy 2010; 65 (Suppl. 1): 3-17.

14. Kojima A, et al. Extended-spectrum-beta-lactamaseproducing Escherichia coli strains isolated from farm animals from 1999 to 2002: report from the Japanese Veterinary Antimicrobial Resistance Monitoring Program. Antimicrobial Agents and Chemotherapy 2005; 49: 3533-3537.

15. Rogers BA, Sidjabat HE, Paterson DL. Escherichia coli O25b-ST131: a pandemic, multiresistant, communityassociated strain. Journal of Antimicrobial Chemotherapy 2011; 66: 1-14.

16. Moreno A, et al. Extended-spectrum beta-lactamases belonging to CTX-M group produced by Escherichia coli strains isolated from companion animals treated with enrofloxacin. Veterinary Microbiology 2008; 129: 203-208.

17. Rossi F. The challenges of antimicrobial resistance in Brazil. Clinical Infectious Diseases 2011; 52: 1138-1143.

18. Baquero F, et al. In vitro susceptibilities of aerobic and facultatively anaerobic gram-negative bacilli isolated from patients with intra-abdominal infections worldwide: 2005 results from Study for Monitoring Antimicrobial Resistance Trends (SMART). Surgical Infections (Larchmont) 2009; 10: 99-104.

19. Andrade SS, et al. Increased resistance to first-line agents among bacterial pathogens isolated from urinary tract infections in Latin America: time for local guidelines? Memórias do Instituto Oswaldo Cruz 2006; 101: 741-748.

20. Bours PH, et al. Increasing resistance in communityacquired urinary tract infections in Latin America, five years after the implementation of national therapeutic guidelines. International Journal of Infectious Diseases 2010; 14: e770-e774.

21. Casellas JM; Antimicrobial Resistance Committee. Results of the 7th Survey of the Antimicrobial Resistance Committee of the Pan American Association of Infectious Diseases. Revista Panamericana de Infectología 2006; 8: 48-55.

22. Alvarez E, Espino M, Contreras R. Antimicrobial susceptibility of Escherichia coli urinary tract isolates by the DIRAMIC system. Revista Panamericana de Infectología 2006; 8: 10-15.

23. Gales AC, Sader HS, Jones RN. Urinary tract infection trends in Latin American hospitals: report from the SENTRY antimicrobial surveillance program (19972000). Diagnostic Microbiology \& Infectious Disease 2002; 44: 289-299.

24. Guzman-Blanco M, Casellas JM, Sader HS. Bacterial resistance to antimicrobial agents in Latin America. The giant is awakening. Infectious Disease Clinics of North America 2000; 14: 67-81.

25. Zurita J. Urinary E. coli: resistance trend in Ecuador from 1999 to 2007. Revista Ecuatoriana de Ginecologia y Obstetricia 2009; 15: 29-35.

26. Pitout JD, Laupland KB. Extended-spectrum betalactamase-producing Enterobacteriaceae: an emerging public-health concern. Lancet Infectious Diseases 2008; 8: 159-166.
27. Rodríguez-Baño J, et al. Bacteremia due to extendedspectrum beta-lactamase-producing Escherichia coli in the CTX-M era: a new clinical challenge. Clinical Infectious Diseases 2006; 43: 1407-1414.

28. O'Brien TF, Stelling J. Integrated multilevel surveillance of the world's infecting microbes and their resistance to antimicrobial agents. Clinical Microbiology Reviews 2011; 24: 281-295.

29. Bradford PA. Extended-spectrum beta-lactamases in the 21st century: characterization, epidemiology, and detection of this important resistance threat. Clinical Microbiology Reviews 2001; 14: 933-951.

30. Minarini LA, et al. Prevalence of community-occurring extended spectrum beta-lactamase-producing Enterobacteriaceae in Brazil. Current Microbiology 2007; 54: 335-341.

31. Cantón R, Coque TM. The CTX-M beta-lactamase pandemic. Current Opinion in Microbiology 2006; 9: 466- 475.

32. Mattar S, Martinez P. Emergence of antimicrobial resistance to extended-spectrum- $\beta$-lactamases (ESBL): detection, clinic impact and epidemiology. Infectio 2007; 11: 23-35.

33. Cantón R, González-Alba JM, Galán JC. CTX-M enzymes: origin and diffusion. Frontiers in Microbiology 2012; 3: 110.

34. Ben-Ami R, et al. A multinational survey of risk factors for infection with extended-spectrum beta-lactamaseproducing Enterobacteriaceae in nonhospitalized patients. Clinical Infectious Diseases 2009; 49: 682-690.

35. Casellas JM. Antibacterial drug resistance in Latin America: consequences for infectious disease control. Revista Panamericana de Salud Pública 2011; 30: 519-528.

36. Hawser SP, et al. Epidemiologic trends, occurrence of extended-spectrum beta-lactamase production, and performance of ertapenem and comparators in patients with intra-abdominal infections: analysis of global trend data from 2002-2007 from the SMART study. Surgical Infections (Larchmont) 2010; 11: 371-378.

37. Rocha JL, Tuon FF, Johnson JR. Sex, drugs, bugs, and age: rational selection of empirical therapy for outpatient urinary tract infection in an era of extensive antimicrobial resistance. Brazilian Journal of Infectious Diseases 2012; 16: 115-121.

38. Pan-American Health Organization. Annual Report of the Network of Monitoring/Surveillance of Antimicrobial Resistance. Lima, Peru, 3 and 4 December 2009. (http://new.paho.org/hq/index.php?option=com_docman\& task=doc_view\&gid=14876\&Itemid=3938).

39. Pan-American Health Organization. Annual Report of the Latin American Network for Surveillance of Antimicrobial Resistance. San José, Costa Rica, 29-30 November, 1 December, 2010.

40. Gordillo MR, Mejía CR. Analysis of resistance of urinary cultures 2005-2010, Roosevelt Hospital, Guatemala City. Revista del Colegio de Medicos y Cirujanos de Guatemala 2011; VI: 51-56.

41. Silva FO, et al. Results of antimicrobial susceptibility surveillance in Chile: consolidating a network. Revista Chilena de Infectología 2011; 28: 19-27. 
42. Villegas MV, et al. Increasing prevalence of extendedspectrum-beta-lactamase among Gram-negative bacilli in Latin America-2008 update from the Study for Monitoring Antimicrobial Resistance Trends (SMART). Brazilian Journal of Infectious Diseases 2011; 15: 34-39.

43. Hawser SP, et al. Low frequency of ertapenem-resistant intra-abdominal isolates of Escherichia coli from Latin America: susceptibility, ESBL-occurrence, and molecular characterisation (SMART 2008-2009). Journal of Chemotherapy 2012; 24: 6-11.

44. van der Starre WE, et al. Risk factors for fluoroquinolone-resistant Escherichia coli in adults with community-onset febrile urinary tract infection. Journal of Antimicrobial Chemotherapy 2011; 66: 650-656.

45. Cohen-Nahum K, et al. Urinary tract infections caused by multi-drug resistant Proteus mirabilis: Risk factors and clinical outcomes. Infection 2010; 38: 41-46.

46. Wu UI, et al. Risk factors for bloodstream infections due to extended-spectrum beta-lactamase-producing Escherichia coli. Journal of Microbiology, Immunology and Infection 2010; 43: 310-316.

47. Cassier P, et al. Cephalosporin and fluoroquinolone combinations are highly associated with CTX-M beta-lactamase-producing Escherichia coli: a casecontrol study in a French teaching hospital. Clinical Microbiology and Infection 2011; 17: 1746-1751.

48. Paterson DL, et al. International prospective study of Klebsiella pneumoniae bacteremia: implications of extended-spectrum beta-lactamase production in nosocomial infections. Annals of Internal Medicine 2004; 140: 26-32.

49. Saely S, et al. Investigating the impact of the definition of previous antibiotic exposure related to isolation of extended spectrum beta-lactamase-producing Klebsiella pneumoniae. American Journal of Infection Control 2011; 39: 390-395.

50. Lahey Clinic. OXA-type beta-lactamases (http:// www.lahey.org/studies/other.asp). Accessed 13 March 2013.

51. Mendes RE, et al. Ability of Latin America laboratories to detect antimicrobial resistance patterns: experience of the SENTRY Antimicrobial Surveillance Program (1997-2000). Brazilian Journal of Infectious Diseases 2003; 7: 282-289.

52. Cormican MG, Marshall SA, Jones RN. Detection of extended-spectrum beta-lactamase (ESBL)-producing strains by the Etest ESBL screen. Journal of Clinical Microbiology 1996; 34: 1880-1884.

53. Clinical and Laboratory Standards Institute. Performance standards for antimicrobial susceptibility testing (2010). M100-S20. CLSI, Wayne, PA.

54. National Committee for Clinical Laboratory Standards. Performance standards for antimicrobial susceptibility testing. 12th informational supplement. M100-S12. Wayne, PA: National Committee for Clinical Laboratory Standards, 2002.

55. Wiegand I, et al. Detection of extended-spectrum beta-lactamases among Enterobacteriaceae by use of semiautomated microbiology systems and manual detection procedures. Journal of Clinical Microbiology 2007; 45: 1167-1174.

56. Manges AR, et al. Endemic and epidemic lineages of Escherichia coli that cause urinary tract infections. Emerging Infectious Disease Journal 2008; 14: 1575-1583.

57. Coque TM, et al. Dissemination of clonally related Escherichia coli strains expressing extended-spectrum beta-lactamase CTX-M-15. Emerging Infectious Diseases 2008; 14: 195-200.

58. Pitout JD, et al. Molecular characteristics of extendedspectrum-beta-lactamase-producing Escherichia coli isolates causing bacteremia in the Calgary Health Region from 2000 to 2007: emergence of clone ST131 as a cause of community-acquired infections. Antimicrobial Agents and Chemotherapy 2009; 53: 2846-2851.

59. Leal AL, et al. Emergence of resistance to third generation cephalosporins by Enterobacteriaceae causing community-onset urinary tract infections in hospitals in Colombia. Enfermedades Infecciosas y Microbiologia Clinica. Published online: 14 June 2012. doi.org/ 10·1016/j.eimc.2012·04·007.

60. Peirano G, et al. Molecular characteristics of extendedspectrum beta-lactamase-producing Escherichia coli from Rio de Janeiro, Brazil. Clinical Microbiology and Infection 2011; 17: 1039-1043.

61. Ruiz SJ, et al. First characterization of CTX-M15-producing Escherichia coli ST131 and ST405 clones causing community-onset infections in South America. Journal of Clinical Microbiology 2011; 49: 1993-1996.

62. Martinez P, Garzón D, Mattar S. CTX-M-producing Escherichia coli and Klebsiella pneumoniae isolated from community-acquired urinary tract infections in Valledupar, Colombia. Brazilian Journal of Infectious Diseases 2012; 16: 420-425.

63. Dias RC, et al. Clonal composition of Escherichia coli causing community-acquired urinary tract infections in the State of Rio de Janeiro, Brazil. Microbial Drug Resistance 2009; 15: 303-308.

64. Queiroz ML, et al. Characterization of extendedspectrum beta-lactamases, antimicrobial resistance genes, and plasmid content in Escherichia coli isolates from different sources in Rio de Janeiro, Brazil. Diagnostic Microbiology \& Infectious Disease 2012; 74: 91-94.

65. Millán B, et al. CTX-M-14 beta-lactamase-producing Citrobacter freundii isolated in Venezuela. Annals of Clinical Microbiology and Antimicrobials 2011; 10: 22.

66. Gupta K, et al. International clinical practice guidelines for the treatment of acute uncomplicated cystitis and pyelonephritis in women: A 2010 update by the Infectious Diseases Society of America and the European Society for Microbiology and Infectious Diseases. Clinical Infectious Diseases 2011; 52: e103-120.

67. Center for Disease Dynamics Economics \& Policy (CDDEP). Exploring ResistanceMap: the rise of fluoroquinolone resistance. Available online at: http://www. cddep.org/blog/posts/exploring_resistancemap_rise_ fluoroquinolone_resistance_part_1. Accessed 10 January 2013. 